\title{
The new cardioprotector Monohydroxyethylrutoside protects against doxorubicin-induced inflammatory effects in vitro
}

\author{
MAI Abou El Hassan*,', HMW Verheul', AS Jorna', C Schalkwijk ${ }^{2}$, J van Bezu², WJF van der Vijgh ${ }^{2}$ and A Bast ${ }^{3}$ \\ 'Department of Medical Oncology, Free University Medical Center, PO Box 7057, 1007 MB Amsterdam, The Netherlands; ${ }^{2}$ Department of Clinical \\ Chemistry, Free University Medical Center, PO Box 7057, 1007 MB Amsterdam, The Netherlands; ${ }^{3}$ Department of Pharmacology and Toxicology, \\ University of Maastricht, PO Box 616, 6200 MD Maastricht, The Netherlands
}

\begin{abstract}
Besides its cardiotoxic effect, doxorubicin also elicits inflammatory effects in vivo. 7-Monohydroxyethylrutoside (monoHER) has recently been used as a protector against doxorubicin-induced cardiotoxicity in vivo. It is not known yet whether monoHER can also protect against doxorubicin-induced inflammatory effects. The aim of the present study was (I) to illustrate the inflammatory effects of doxorubicin in vitro and (2) to evaluate a possibly protective effect of monoHER. In order to demonstrate the inflammatory effects of doxorubicin and the possible protection of monoHER, proliferating human umbilical cord vascular endothelial cells (HUVECs) were incubated with different concentrations of doxorubicin ranging from 12.5 to $600 \mathrm{nM}$ with(out) $200 \mu \mathrm{M}$ monoHER. Resting (confluent) HUVECs were incubated with $(0.5-25 \mu \mathrm{M})$ doxorubicin with(out) monoHER $(0.2-1.2 \mathrm{~mm})$ and the viability of endothelial cells and their propensity to adhere to neutrophils were measured $24 \mathrm{~h}$ after treatment. The localisation of adhered neutrophils was determined with immunofluorescence microscopy. To further characterise the mechanism of doxorubicin-induced neutrophil adhesion, the expression of the HUVECs surface adhesion molecules was determined after doxorubicin treatment. Doxorubicin decreased the viability and proliferation capacity of HUVECs in a concentration-dependent manner. The proliferating HUVECs were much more sensitive to doxorubicin $\left(\mathrm{IC}_{50}=60.0 \pm 20.8 \mathrm{nM}\right)$ than resting cells $\left(\mathrm{LC}_{50}=4.0 \pm 0.3 \mu \mathrm{M}\right)$. Doxorubicin also increased the adhesion of neutrophils reaching a plateau value at a doxorubicin concentration of $\geqslant 0.4 \mu \mathrm{M}(P=0.0 \mathrm{I} \mid 3)$. The induced neutrophil adhesion was accompanied by overexpression of VCAM and E-selectin but not ICAM. Although monoHER did not reverse the effect of doxorubicin on the proliferation of endothelial cells, it significantly protected resting HUVECs against the cytotoxic effect of doxorubicin ( $\leqslant 25 \mu \mathrm{M}, P<0.0015$ ). In addition, monoHER completely protected against the stimulatory effect of doxorubicin on neutrophil adhesion, and inhibited the doxorubin-induced expression of VCAM and E-selectin on the surface of treated HUVECs. This study illustrates that monoHER, which protects against doxorubicin's cardiotoxic effect, can also protect against doxorubicin-induced inflammatory effects. These data prompt further investigation about the possible link between doxorubicin-induced inflammatory effects and its cardiotoxicity in vivo.

British Journal of Cancer (2003) 89, 357-362. doi:I0.1038/sj.bjc.660I022 www.bjcancer.com
\end{abstract}

(c) 2003 Cancer Research UK

Keywords: doxorubicin; monoHER; HUVECs; MTT; neutrophil adhesion

Doxorubicin is a well-known chemotherapeutic agent used against a wide range of human malignancies. The development of acute and chronic cardiotoxicity (Schwartz et al, 1993), however, limits the clinical use of doxorubicin. The mechanism of doxorubicininduced cardiotoxicity is still not fully understood. The prevailing hypothesis, however, suggests that the cardiotoxicity results from the formation of reactive oxygen species (ROS) (e.g. hydroxyl $\left(\mathrm{OH}^{*}\right)$ and superoxide anion $\left(\mathrm{O}_{2}^{-*}\right)$ radicals) (Olson and Mushlin, 1990; Takacs et al, 1992; Goeptar et al, 1994). Reactive oxygen species affect heart tissue specifically because of its relatively low antioxidants content (e.g. SOD and catalase) and the relative abundance of mitochondria (Takacs et al, 1992).

In addition to its cardiotoxic effects, doxorubicin elicits also inflammatory effects as illustrated by the occurrence of phlebitis

*Correspondence: Dr MAl Abou El Hassan;

E-mail: m.abulhassan@vumc.nl

Revised 7 April 2003; accepted 13 April 2003
(Hecker, 1990) and acute inflammatory reactions in the eyelid of different laboratory animals (McLoon and Wirtschafter, 1997). Doxorubicin also causes inflammatory reactions in the vicinity of heart tissue where it was found to increase the incidence of thrombus formation in the atrium of mice (Fujihira et al, 1993).

Recently, we have shown that 7-monohydroxyethylrutoside (monoHER) could protect against doxorubicin-induced cardiotoxicity in the mouse (Van Acker et al, 2000; Van Acker et al, 1997). MonoHER cardioprotection is believed to result from the protection against doxorubicin-induced free radicals as a good antioxidant and metal ion chelating agent (Haenen et al, 1993; Van Acker et al, 1993). Since the mechanism of monoHER is not fully established, it is also important to study the effect of monoHER on the inflammatory effects of doxorubicin.

Accordingly, the aim of this study was to establish a model showing the inflammatory effects of doxorubicin in vitro. Using this model, the effect of doxorubicin on the viability and proliferating capacity of human vascular endothelial Cells (HUVECs) was studied. Furthermore, the effect of doxorubicin 
on the neutrophil adhesion of HUVECs was studied. In the mean time, the expression of several adhesion molecules on the surface of doxorubicin-treated HUVECs was also studied. The study was completed by investigating the protection obtained by monoHER against the inflammatory effects of doxorubicin in that model.

\section{MATERIALS AND METHODS}

\section{Chemicals}

7-monohydroxyethylrutoside was kindly provided by Novartis Consumer Health (Nyon, Switzerland). Doxorubicin $\mathrm{HCl}$ was purchased from Pharmacia Upjohn BV (Woerden, The Netherlands). Potassium chloride, sodium chloride, ammonium chloride, EDTA (disodium salt), potassium bicarbonate and dimethyl sulphoxide (DMSO) were purchased from Merck (Amsterdam, The Netherlands), bovine serum albumin, trypsin, 3-(4,5-dimethylthiazol-2-yl)-2,5-diphenyltetrazolium bromide (MTT) and phenyl methyl sulphonyl chloride (PMSF) from Sigma-Aldrich Chemie (Zwijndrecht, The Netherlands), D-glucose and nonidet P40 (NP40) from Fluka Biochemika (Buchs, Switzerland), human serum from CLB (Amsterdam, The Netherlands) and M199, fetal calf serum (FCS) and HBSS (without calcium) from Life Technologies (Breda, The Netherlands). Calcein AM was from Molecular Probes Europe BV (Leiden, The Netherlands), Ficoll from Pharmacia Biotech AB (Upssala, Sweden) and tumour necrosis factor $\alpha$ (TNF $\alpha$ ), L-glutamine, fibronectin (human plasma), heparin and HEPES from ICN Biomedicals (Aurora, $\mathrm{OH})$. Endothelial cell growth factor (ECGF) was extracted from bovine hypothalamus as previously mentioned (Maciag et al, 1979).

\section{Human vascular endothelial cells isolation and culture}

Human vascular endothelial cells were isolated as previously described (Verheul et al, 2000). In brief, the umbilical cord vein was filled with trypsin/EDTA, after being washed with warm PBS, and incubated at $37^{\circ} \mathrm{C}$ for $20 \mathrm{~min}$. The dissociated cells were collected by washing the vein with PBS and the cell suspension was centrifuged at $241 \mathrm{~g}$ for $7 \mathrm{~min}$. The cell pellet was quickly resuspended in culture medium (M199 containing 10\% human serum, $10 \% \mathrm{FCS}, 5 \mathrm{U} \mathrm{ml}^{-1}$ heparin, $200 \mathrm{IE} \mathrm{ml}^{-1}$ penicillin and $200 \mu \mathrm{g}$ streptomycin, $0.29 \mathrm{mg} \mathrm{ml}^{-1}$ L-glutamine and $50 \mu \mathrm{g} \mathrm{ml}^{-1}$ ECGF). Endothelial cells passage 2 (P2), plated in fibronectinprecoated 96-well flat-bottom microtitre plate (Costar, Badhoevedorp, The Netherlands), was used during the whole study.

\section{Proliferation assay $\left(\mathrm{IC}_{50}\right)$}

Human vascular endothelial cells were plated on precoated 96-well plate with a density of 3000 cells/well. Cells were incubated with different concentrations of doxorubicin $(12.5-600 \mathrm{nM}$, prepared in culture medium) with(out) $(200 \mu \mathrm{M})$ monoHER. Dimethyl sulphoxide was used as a solubiliser for monoHER with a final concentration of $0.1 \%$, which did not show any antiproliferative effect. Cells were washed $(3 \times)$ with warm medium $24 \mathrm{~h}$ after treatment and were left to grow for another $48 \mathrm{~h}$. Thereafter, the MTT assay was performed according to Mosmann (1983) with minor modifications. The percentage growth (proliferation) was calculated by dividing the increase in the cell count (after the time of the experiment, i.e. 3 days) of the treated cells by the increase of the cell count of the untreated cells (which was taken as $100 \%$ proliferation). The decrease in the proliferation capacity (as percentage of control) was plotted as a function of the doxorubicin concentration. The $\mathrm{IC}_{50}$ of doxorubicin with(out) monoHER was estimated from the plot.

\section{Cytotoxicity assay $\left(\mathrm{LC}_{50}\right)$}

In the cytotoxicity studies confluent HUVECs were used. The $\mathrm{LC}_{50}$ was determined by treating confluent cells with different concentrations of doxorubicin $(0.5-25 \mu \mathrm{M})$ with(out) monoHER $(0.2-1.2 \mathrm{~mm})$. The viability was measured $24 \mathrm{~h}$ after treatment using the MTT assay. Dimethyl sulphoxide, used as a solubilizer for monoHER, reached a final concentration of $0.5 \%$, which hardly decreased (5\%) the viability of confluent HUVECs. The percentage viability (taking the control as $100 \%$ viability) was plotted as a function of doxorubicin or monoHER concentration. The $\mathrm{LC}_{50}$ of doxorubicin with(out) monoHER was estimated.

\section{Isolation and preparation of calcein-labelled human neutrophils}

Isolation of neutrophils was performed as previously reported (Schaefer et al, 1998) with a slight modification. In short, $24 \mathrm{ml}$ of venous blood was drawn, by venipuncture, from healthy donors into vacutainer tubes (Becton Dickinson, Plymouth, UK) containing $0.11 \mathrm{M}$ sodium citrate and centrifuged at $1500 \mathrm{~g}$ for $5 \mathrm{~min}$. The buffy coat was carefully layered over a Ficoll solution and was centrifuged at $540 \mathrm{~g}$ for $20 \mathrm{~min}$ at room temperature. Neutrophils were isolated from the red pellet after the complete lysis of the erythrocytes (with lysis buffer containing $0.15 \mathrm{M} \mathrm{NH}_{4} \mathrm{Cl}, 1 \mathrm{~mm}$ $\mathrm{KHCO}_{3}$ and $0.1 \mathrm{M} \mathrm{Na} \mathrm{NDDA}_{2}$ ) at $0{ }^{\circ} \mathrm{C}$ followed by centrifugation at $380 \mathrm{~g}$ for $5 \mathrm{~min}$ at $4^{\circ} \mathrm{C}$. After counting, the cell density was adjusted to $2.5 \times 10^{6} \mathrm{cell} / \mathrm{ml}$ in M199 medium. Neutrophils were labelled by incubation with $2.5 \mu \mathrm{M}$ of the florescent indicator calcein AM in the dark for $15 \mathrm{~min}$ at $37^{\circ} \mathrm{C}$.

\section{Neutrophil adhesion assay}

Confluent HUVECs were incubated for $24 \mathrm{~h}$ with different concentrations (range $0.2-1.0 \mu \mathrm{M}$ ) of doxorubicin, prepared in M199 containing 10\% human serum, alone or in combination with $1 \mathrm{mM}$ of monoHER (dissolved in $0.5 \%$ DMSO-containing medium). Dimethyl sulphoxide did not affect the adhesion of neutrophils to HUVECs in vitro. After stimulation, HUVECs were washed with prewarmed M199 (2X). $100 \mu \mathrm{l}$ of the calcein-labelled neutrophils was added per well and left to adhere for $1 \mathrm{~h}$ at $37^{\circ} \mathrm{C}$. Subsequently, the unbound neutrophils were thoroughly washed away with warm PBS (3X). The fluorescent dye was released by lysis of the bound neutrophils with $50 \mu \mathrm{l}$ of lysis buffer containing $1 \%$ NP40, $150 \mathrm{~mm}$ $\mathrm{NaCl}, 10 \mathrm{~mm}$ Tris- $\mathrm{HCl}$ (pH 7.5), $5 \mathrm{~mm}$ EDTA and $1 \mathrm{~mm}$ PMSF. After $10 \mathrm{~min}$ of shaking, the fluorescence was measured with a spectrafluor multiplate reader TECAN (Salzburg, Austria) with excitation and emission filters of 492 and $535 \mathrm{~nm}$, respectively. The percentage adhesion (taking the control as zero percent adhesion) was plotted as a function of doxorubicin concentration.

For the validation of the procedure, TNF $\alpha\left(1000 \mathrm{U} \mathrm{ml}^{-1}\right)$ was used as a positive control. As previously reported (Clark et al, 1997) TNF $\alpha$ stimulation caused an increase in neutrophil adhesion of HUVECs with a factor of 2.5-4 compared to the blank.

\section{Immunofluorescence microscopy}

To localise neutrophil adhesion to doxorubicin-stimulated HUVECs, bound neutrophils were not lysed but fixed with $4 \%$ paraformaldehyde for $15 \mathrm{~min}$. After washing, cells were incubated with $10 \%$ FCS and $0.02 \mathrm{M}$ glycine in PBS for $30 \mathrm{~min}$ followed by incubation with the primary antibodies CD 15 FITC $(1: 40$ dilution) (Becton Dickinson, Leiden, The Netherlands) and biotinylated ulex europaeus agglutinin 1 ( $1: 100$ dilution) (Vector Laboratories, Inc., Burlingame, CA, USA) for the neutrophils and the endothelial cells, respectively. After washing with PBS (3X), streptavidin-Texas red (Vector Lab., Inc.), as fluorescent labelled secondary antibody, was added (1:100 dilution) for the detection 
of the biotinylated lectin. After washing, the wells were carefully dried and mounted with Vectashield mounting medium for fluorescence (H1200, Vector Lab. Inc.). Stained wells were examined with an, LEICA confocal microscope showing neutrophils in green and HUVECs in red. A rarely appearing yellow colour represented areas of coincident labelling with both antibodies.

\section{Expression of cell surface adhesion molecules}

Confluent HUVECs, cultured in M199 containing 10\% human serum, were incubated for $24 \mathrm{~h}$ with different concentrations (range $0.08-1.0 \mu \mathrm{M}$ ) of doxorubicin alone or in combination with $1 \mathrm{mM}$ of monoHER (dissolved in $0.13 \%$ DMSO-containing medium). Dimethyl sulphoxide did not affect the expression of the cell surface VCAM, ICAM or E-selectin. Under these conditions, the positive control, that is, cells treated with TNF $\alpha$ $\left(10 \mathrm{U} \mathrm{ml}^{-1}\right)$, induced between three- and five-fold increase in the expression of all the adhesion molecules.

The expression of cell-bound VCAM-1, ICAM-1 and E-selectin was measured as described earlier (Carlos et al, 1990). The colour intensity was measured at a wavelength of $450 \mathrm{~nm}$. Experiments were performed in triplicate. The results were expressed as percentage of the control (taking the control as zero control), which was plotted as a function of doxorubicin concentration.

\section{Statistical analysis}

The results are expressed as the mean value of three different days ( \pm s.e.m.). The value of each day represents the mean value of three wells. The cells used on each day were obtained from a separate umbilical cord. The overall effect of doxorubicin with(out) monoHER was evaluated with ANOVA. When the ANOVA indicated significant effect, the contrasts (differences) of the effect of each concentration from the blank were investigated. For monoHER protection, the contrast between monoHER effect at each doxorubicin concentration (viz. the difference between doxorubicin + monoHER and doxorubicin alone) and monoHER alone (viz. taking the change due to monoHER alone into account) was also investigated.

The comparison of the $\mathrm{IC}_{50}$ or the $\mathrm{LC}_{50}$ of doxorubicin alone or with monoHER was made using student's $t$-test.

\section{RESULTS}

\section{Antiproliferative effect of doxorubicin $\left(\mathrm{IC}_{50}\right)$}

The antiproliferative effect of doxorubicin on HUVECs is shown in Figure 1. Doxorubicin decreased the proliferation of endothelial cells in a concentration-dependent manner and the $\mathrm{IC}_{50}$ was $60 \pm 21 \mathrm{~nm}$ after $24 \mathrm{~h}$. Coincubation with $200 \mu \mathrm{m}$ monoHER did not significantly change the $\mathrm{IC}_{50}$ of doxorubicin $(93 \pm 54 \mathrm{nM})$.

\section{Cytotoxic effect of doxorubicin $\left(\mathrm{LC}_{50}\right)$}

In this assay, confluent HUVECs were used to exclude proliferation of the endothelial cells. Confluent HUVECs were 65-fold more resistant to doxorubicin compared to proliferating cells and the $\mathrm{LC}_{50}$ was $4.0 \pm 0.3 \mu \mathrm{M}$.

The protective effect of different concentrations of monoHER against $5 \mu \mathrm{M}$ doxorubicin (which is almost equal to the $\mathrm{LC}_{50}$ of doxorubicin) is shown in Figure 2. Forty percent of HUVECs remained viable after incubation with $5 \mu \mathrm{M}$ doxorubicin for $24 \mathrm{~h}$. 7Monohydroxyethlrutoside $(0.2-1.2 \mathrm{~mm})$ alone did not affect the viability of confluent HUVECs. Human vascular endothelial cells were significantly protected against the cytotoxic effect of doxorubicin at monoHER concentrations $\geqslant 0.8 \mathrm{mM}(P=0.0014)$ compared to doxorubicin alone. Maximum protection (about $30 \%$

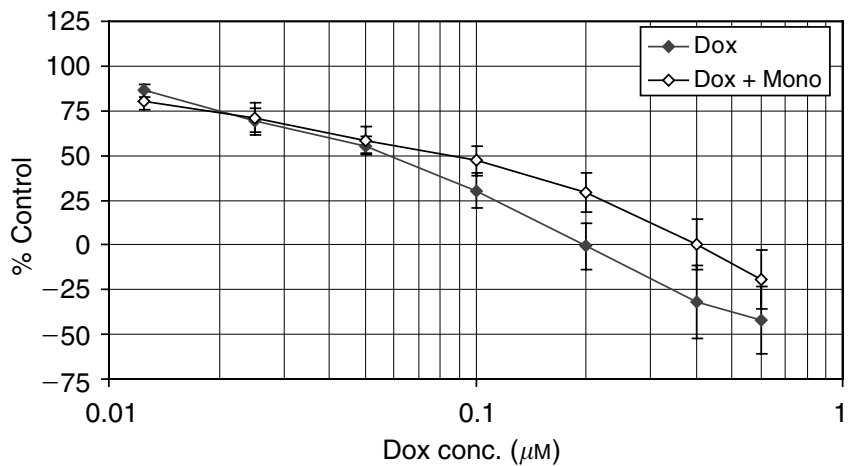

Figure I The effect of doxorubicin with(out) monoHER on the proliferation of HUVECs $24 \mathrm{~h}$ after treatment compared to control. Values are mean $(n=3) \pm$ s.e.m.

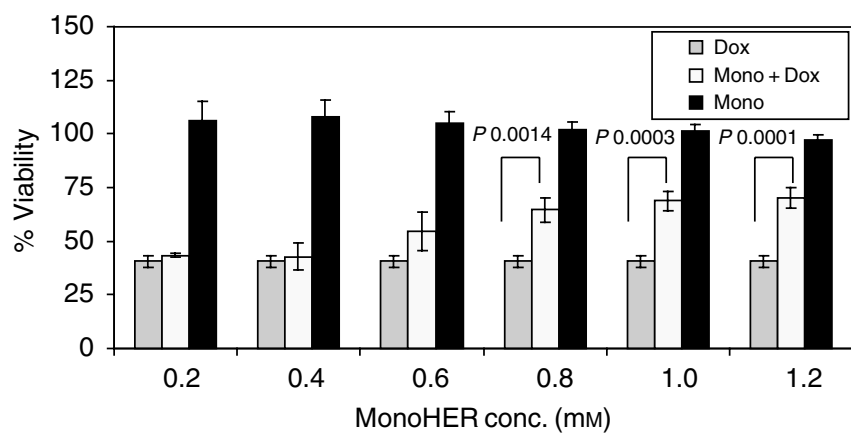

Figure 2 The viability of endothelial cells $24 \mathrm{~h}$ after treatment with $5 \mu \mathrm{M}$ doxorubicin with(out) different concentrations of monoHER. Values are mean $(n=3)+$ s.e.m. The $P$ values, indicating monoHER concentrations that significantly protected against the damaging effect of $(5 \mu \mathrm{M})$ doxorubicin, were calculated with ANOVA.

of the viability) was attained when a monoHER concentration of $\geqslant 1 \mathrm{~mm}(P=0.0003)$ was used.

Figure 3 shows the viability of confluent HUVECs $24 \mathrm{~h}$ after exposure to different concentrations of doxorubicin with(out) $1 \mathrm{~mm}$ monoHER. Doxorubicin significantly decreased the viability of confluent HUVECs in a concentration-dependent manner $(P<0.00005)$. Coincubation with $1 \mathrm{~mm}$ monoHER protected endothelial cells against doxorubicin and the $\mathrm{LC}_{50}$ of doxorubicin significantly increased from $4 \pm 0.3$ to $40 \pm 10 \mu \mathrm{M}(P<0.05)$. 7Monohydroxyethykrutoside protection was dependent on the doxorubicin concentration: maximum protection was obtained when the doxorubicin concentration was $5 \mu \mathrm{M}(P<0.00005)$ and significant protection could still be obtained when doxorubicin concentrations were $\leqslant 25 \mu \mathrm{M}(P=0.0015)$.

\section{Immunofluorescence microscopy}

Immunofluorescence microscopy was used for the localization of adhering neutrophils upon stimulation with doxorubicin with(out) monoHER. As a result of the cellular damage induced by doxorubicin, concentrations of $\leqslant 1 \mu \mathrm{M}$ were used in order to reduce the nonspecific adhesion of neutrophils (i.e. to the fibronectin-coated bottom). Figure 4 shows the adhesion of neutrophils (in green) to HUVECs (in red) after stimulation with $0.6 \mu \mathrm{M}$ doxorubicin with(out) $1 \mathrm{~mm}$ monoHER in comparison to unstimulated cells (blank). Few neutrophils were bound to unstimulated HUVECs. Neutrophil adhesion increased by stimulation of HUVECs with $0.6 \mu \mathrm{m}$ doxorubicin. Neutrophils were 


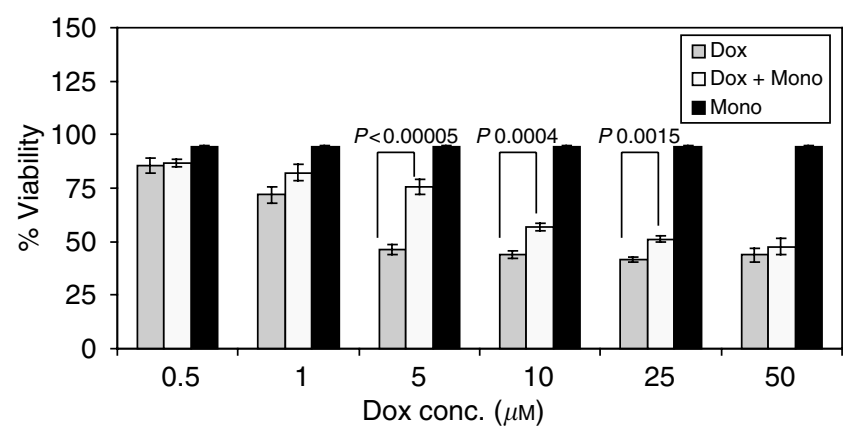

Figure 3 The viability of endothelial cells $24 \mathrm{~h}$ after treatment with different concentrations of doxorubicin with(out) I mM monoHER. Values are mean $(n=3)+$ s.e.m. The $P$ values, which indicate the significant protection of monoHER against the damaging effect of different concentrations of doxorubicin, were calculated with ANOVA.
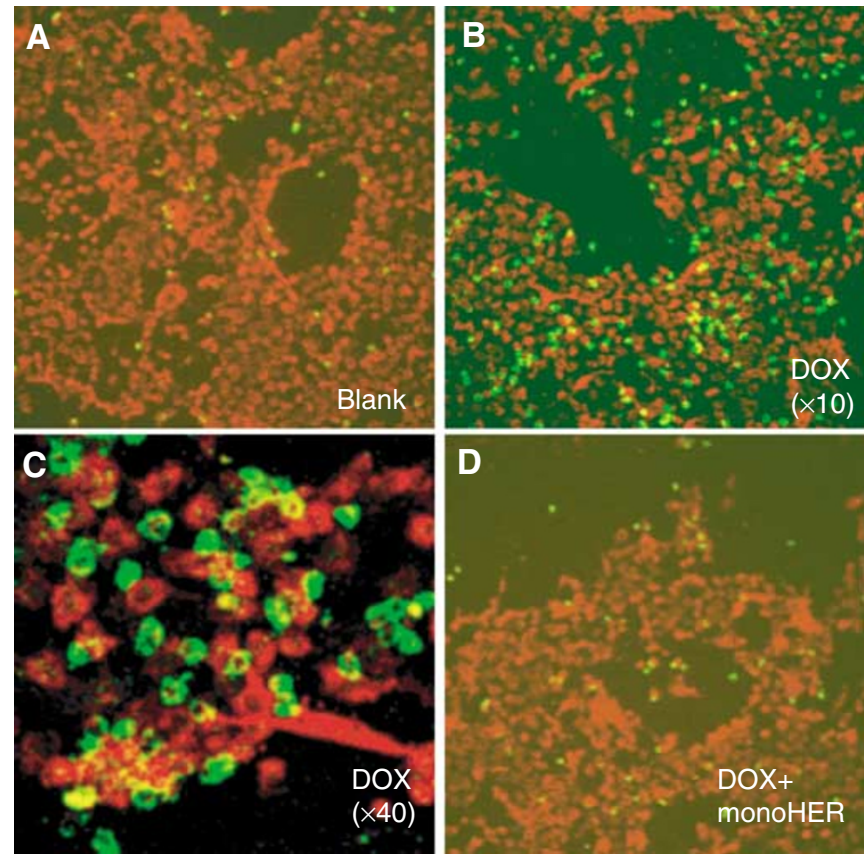

Figure 4 Immunofluorescence microscopy of neutrophil (in green) adhesion after stimulation with doxorubicin $(0.6 \mu \mathrm{M})$ with (out) I mM monoHER compared to unstimulated HUVECs (in red).

specifically bound to the endothelial cells as shown in Figure $4 \mathrm{C}$ $(\times 40)$. Coincubation with monoHER completely reverted doxorubicin-induced neutrophil adhesion back to basal levels (Figure 4D).

\section{Adhesion of neutrophils to stimulated HUVECs}

Figure 5 shows the percentage adhesion of neutrophils to confluent HUVECs $24 \mathrm{~h}$ after stimulation with different concentrations of doxorubicin $(0.2-1.0 \mu \mathrm{M})$ alone or in combination with $(1 \mathrm{mM})$ monoHER. Doxorubicin stimulation of endothelial cells resulted in a significant increase in the percentage neutrophil adhesion, as detected by the increase of the fluoresence, compared to the control. This increase was concentration dependent and reached a plateau at doxorubicin concentrations of $\geqslant 0.4 \mu \mathrm{M}(P<0.02)$. 7Monohydroxyethylrutoside alone did not affect the adhesion of neutrophils to HUVECs. Simultaneous incubation with (1 mM)

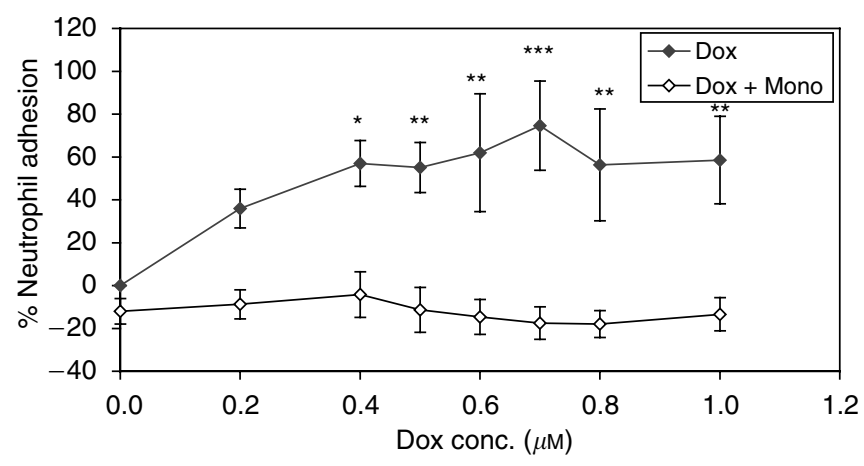

Figure 5 The adhesion of neutrophils to HUVECs (expressed as percentage of the control) was observed $24 \mathrm{~h}$ after stimulation with doxorubicin alone or in combination with $(\mathrm{I} \mathrm{mM})$ monoHER. Values are mean $(n=3) \pm$ s.e.m. The $P$-values $(*<0.02$, *** $<0.0 I$, ***** $<0.00 I$ ), which indicate significant protection of monoHER against doxorubicin-induced neutrophil adhesion of HUVECs, were calculated with ANOVA.

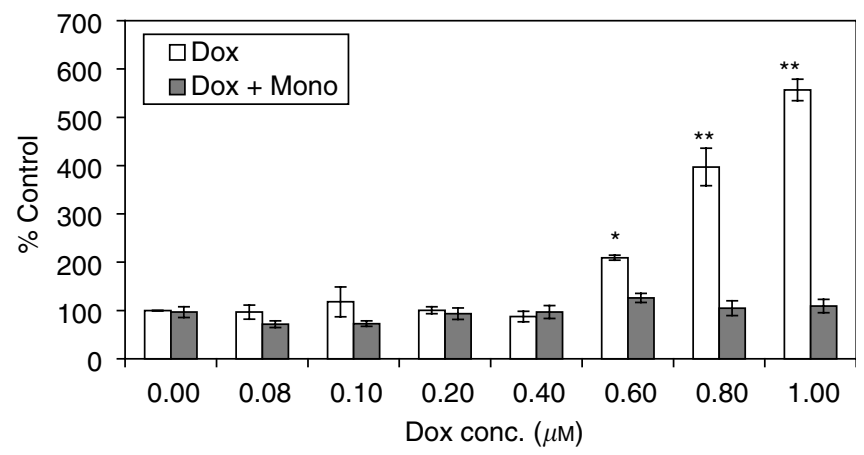

Figure 6 The overexpression of VCAM $24 \mathrm{~h}$ after stimulation with doxorubicin with(out) I mM monoHER. Values are mean $(n=3) \pm$ s.e.m. The $P$ values $(*<0.002$, $* *<0.00 I)$, which indicate significant increase in the expression of VCAM, were calculated with ANOVA.

monoHER reverted doxorubicin-induced adhesion to the basal levels (Figure 5).

\section{Overexpression of cell bound adhesion molecules}

Figures 6 and 7 show the expression of VCAM and E-selectin on the surface of HUVECs, as a percentage from the control, $24 \mathrm{~h}$ after stimulation with different concentrations of doxorubicin with(out) $1 \mathrm{mM}$ monoHER. Both VCAM and E-selectin expression increased significantly $(P<0.002)$ after treatment with doxorubicin concentrations $>0.6$ and $>0.8 \mu \mathrm{M}$, respectively. The expression level of ICAM did not change after the same treatment. The combined treatment with $1 \mathrm{mM}$ monoHER reverted doxorubicin-stimulated overexpression of VCAM and E-selectin to the basal levels.

\section{DISCUSSION}

7-Monohydroxyethylrutoside was recently used in the protection against doxorubicin-induced cardiotoxicity in vivo (Van Acker et al, 1997, 2000). The mechanism of protection is not fully understood. The prevailing hypothesis is scavenging of free radicals produced by doxorubicin and chelation of iron ions (Haenen et al, 1993). Besides cardiotoxic properties, doxorubicin 


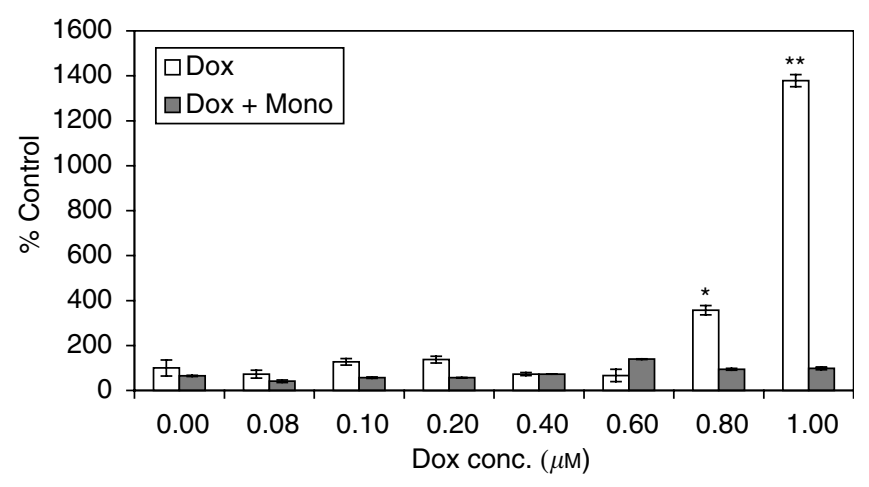

Figure 7 The overexpression of E-selectin $24 \mathrm{~h}$ after stimulation with doxorubicin with(out) I mM monoHER. Values are mean $(n=3) \pm$ s.e.m. The $P$ values $(*<0.001, * *<0.0005)$, which indicate significant increase in the expression of VCAM, were calculated with ANOVA.

has also inflammatory effects. It was therefore thought important to study the role of monoHER against these inflammatory effects.

Thusfar the inflammatory effects of doxorubicin did not have much attention. The inflammatory effects of doxorubicin were not only found in the vasculature as phlebitis (Hecker, 1990) or as skin inflammation after local treatment in the eye lid of several laboratory animals (McLoon and Wirtschafter, 1997) but also in the vicinity of the heart tissue. An increase in neutrophil adhesion and thrombus formation has been found in the atrium of $75 \%$ of mice repeatedly injected with $4 \mathrm{mg} \mathrm{kg}^{-1}$ doxorubicin (Fujihira et al, 1993). It is remarkable that the authors found associated damage in the cardiomyocytes and the presence of interstitial inflammatory cell infiltration. These findings suggest that the inflammatory effect of doxorubicin may be directly or indirectly related to its cardiotoxicity.

In the present study, we have illustrated the inflammatory effects of doxorubicin in vitro. Doxorubicin reduced the viability of resting endothelial cells with concentrations comparable to the concentrations attained in the plasma of human and laboratory animals (Mross et al, 1990; Van der Vijgh et al, 1990). In addition to the direct damage to the vascular endothelial cells, doxorubicin also induced neutrophil adhesion of vascular endothelial cells in vitro.

In order to study the process of neutrophil adhesion to doxorubicin-stimulated HUVECs, the expression of surface-bound adhesion molecules was studied. The present study shows that doxorubicin-induced neutrophil adhesion to stimulated HUVECs was mediated via the overexpression of VCAM and E-selectin but not via ICAM. Furthermore, it also shows that the overexpression of VCAM occurs after stimulation with a lower concentration of doxorubicin $(0.6 \mu \mathrm{M})$ than that for E-selectin $(0.8 \mu \mathrm{M}$ doxorubicin).

Besides the inflammatory effect, the present study has also illustrated that doxorubicin affects proliferating endothelial cells much more markedly ( $\mathrm{IC}_{50}=60 \mathrm{nM}$ ) than resting (confluent) cells $\left(\mathrm{LC}_{50}=4 \mu \mathrm{M}\right)$. Since proliferating endothelial cells are involved in the neovascularisation (angiogenesis), this may indicate that a low dose of doxorubicin may have an antiangiogenic effect without affecting the vascular endothelium (resting endothelial cells with a low turnover rate; Klohs and Hamby, 1999). This finding is supported by Browder et al (2000), who have recently shown the antiangiogenic effect of low-dose chemotherapy.

The present study is the first to show the anti-inflammatory properties of the flavonoid monoHER. The addition of monoHER significantly protected against doxorubicin damaging effect on resting HUVECs in vitro. 7-Monohydroxyethylrutoside also completely inhibited doxorubicin-induced neutrophil adhesion of HUVECs in vitro. This protection may be mediated by the observed inhibition of doxorubicin-induced VCAM and E-selectin overexpression. On the other hand, monoHER did not protect against the antiproliferative effect of doxorubicin.

The anti-inflammatory effect of the flavonoid monoHER could be expected. Different flavonoids have an anti-inflammatory effect, which plays a role in their therapeutic effect on allergy, asthma, viral infection and gastric ulcers (Gerritsen et al, 1995; Havsteen, 1983). The good antioxidant and metal ion chelating properties of monoHER (Haenen et al, 1993; Van Acker et al, 1993) play a role in the protection of endothelial cells against the direct damage of doxorubicin. These properties enable monoHER to protect against doxorubicin-induced oxygen radicals, which induce lysis of the endothelial cells and increase the vascular permeability and reactivity (Clark et al, 1997). In addition, monoHER is one of the constituents of Venoruton ${ }^{\mathbb{R}}$, which is used in the treatment of chronic venous insufficiency by decreasing vascular permeability and protecting vascular endothelial cells (Havsteen, 1983). The protection of monoHER did not interfere with the antiproliferative effect of doxorubicin. This is in line with our previous work where monoHER protected against doxorubicin-induced cardiotoxicity without interfering with its antiproliferative effect both in vitro and in vivo (Van Acker et al, 1997).

The present in vitro study together with several publications, pose an important question about a possible link between doxorubicin-induced inflammatory effects and its cardiotoxicity. Our in vitro data show that doxorubicin affected both the viability and neutrophil adhesion of endothelial cells with clinically achievable concentrations. Others have also shown that doxorubicin has vascular effects in vivo. Doxorubicin was found to disrupt the balance of several agents involved in the regulation of vasodilatation, vasoconstriction and neutrophil adhesion in vivo, for example, endothelin-1 (ET-1), histamine (Decorti et al, 1989, 1997; Duffy et al, 1999) and nitric oxide (NO) (Bristow et al, 1980), leading to an imbalance in the physiology of the vasculature especially after the long-term use of doxorubicin. Besides the vascular imbalance, doxorubicin damage to endothelial cells could cause endothelial dysfunction, which is known to induce myocardial ischaemia, aggravate acute coronary syndromes and accelerate progression of chronic artery disease (Britten and Schachinger, 1998). These observations suggest the occurrence of cardiovascular ailment after doxorubicin administration, which pinpoints to a link between the inflammatory effects of doxorubicin and its induced cardiotoxicity. As a result of the potential clinical applicability, it is important to confirm the link between the inflammatory effects of doxorubicin and its cardiotoxicity in an in vivo model and to study the extent by which these inflammatory effects participate in the development of the heart disease. This can be studied by investigating whether the antiinflammatory effects of monoHER and other anti-inflammatory agents could partially protect against doxorubicin-induced cardiotoxicity in vivo.

\section{CONCLUSION}

Doxorubicin, in clinically relevant concentrations, showed inflammatory effects in vitro (reduced the viability of HUVECs and increased their propensity for neutrophil adhesion via VCAM and E-selectin but not via ICAM). Proliferating endothelial cells were much more sensitive to doxorubicin damage than resting endothelial cells. 7-monohydroxyethylrutoside protected against these inflammatory effects without interfering with the antiproliferative effect of doxorubicin. The possibility of a relation between doxorubicin-induced-inflammatory effects and its cardiotoxicity should be further investigated in vivo. 


\section{REFERENCES}

Bristow MR, Sageman WS, Scott RH, Billingham ME, Bowden RE, Kernoff RS, Snidow GH, Daniels JR (1980) Acute and chronic cardiovascular effects of doxorubicin in the dog: the cardiovascular pharmacology of drug-induced histamine release. J Cardiovasc Pharmacol 2: 487-515

Britten M, Schachinger V (1998) The role of endothelial function for ischemic manifestations of coronary atherosclerosis. Herz 23(2): 97 - 105

Browder T, Butterfield CE, Kraling BM, Shi B, Marshall B, O’Reilly MS, Folkman J (2000) Antiangiogenic scheduling of chemotherapy improves efficacy against experimental drug-resistant cancer. Cancer Res 60(7): $1878-1886$

Carlos TM, Schwartz BR, Kovach NL, Yee E, Rosil M, Osborn L, Chi-Rosso G, Newman B, Lobo R, Bosso M (1990) Vascular cell adhesion molecule-1 mediates lymphocyte adherence to cytokine-activated cultured human endothelial cells. Blood 76: $965-970$

Clark CJ, Boswell F, Greer IA, Lyall F (1997) Treatment of endothelial cells with serum from women with preeclampsia: Effect on neutrophil adhesion. J Soc Gynecol Invest 4: 27-33

Decorti G, Candussio L, Klugmann FB, Strohmayer A, Mucci MP, Mosco A, Baldini L (1997) Adriamycin-induced histamine release from heart tissue in vitro. Cancer Chemother Pharmacol 40: 363-366

Decorti G, Klugmann FB, Candussio L, Furlani A, Scarcia V, Baldini L (1989) Uptake of adriamycin by rat and mouse mast cells and correlation with histamine release. Cancer Res 49: $1921-1926$

Duffy SJ, Castle SF, Harper RW, Meredith IT (1999) Contribution of vasodialator prostanoids and nitric oxide to resting flow, metabolic vasodialation and flow-mediated dialation in human coronary circulation. Circulation 100: 1951 - 1957

Fujihira S, Yamamoto T, Matsumoto M, Yoshizawa K, Oishi Y, Fujii T, Noguchi H, Mori H (1993) The high incidence of atrial thrombosis in mice given doxorubicin. Toxicol Pathol 21(4): 362-368

Gerritsen ME, Carley WW, Ranges GE, Shen C, Phan SA, Ligon GF, Perry C (1995) Flavonoids inhibit cytokine-induced endothelial cell adhesion protein gene expression. Am J Pathol 147: 278-292

Goeptar AR, Groot EJ, Scheerens H, Commandeur JNM, Vermeulen NPE (1994) Cytotoxicity of mitomycin C and adriamycin in freshly isolated rat hepatocytes: the role of cytochrome P450. Cancer Res 54: 2411-2418

Haenen GRMM, Jansen FP, Bast A (1993) The antioxidant properties of five O-(b-hydroxyethyl)-rutosides of the flavonoid mixture Venoruton. Phlebology(Suppl 1): $10-17$

Havsteen B (1983) Flavonoids, a class of natural products of pharmacological potency. Biochem Pharmacol 32: $1141-1148$

Hecker JF (1990) Survival of intravenous chemotherapy infusion sites. $\mathrm{Br} J$ Cancer 62(4): 660-662

Klohs WD, Hamby JM (1999) Antiangiogenic agents. Curr Opin Biotechnol 10(6): $544-549$
Maciag T, Cerundolo J, Ilsely S, Kelley PR, Forand R (1979) An endothelial cell growth factor from bovine hypothalamus: identification and partial characterization. Proc Natl Acad Sci 76(11): $5674-5678$

McLoon LK, Wirtschafter J (1997) Local injections of corticotropin releasing factor reduce doxorubicin-induced acute inflammation in the eyelid. Invest Ophthalmol Vis Sci 38(5): 834-841

Mosmann T (1983) Rapid colorimetric assay for cellular growth and survival: application to proliferation and cytotoxicity assays. J Immunol Methods 65: 55-63

Mross K, Mayer U, Hamm K, Burk K, Hossfeld DK (1990) Pharmacokinetics and metabolism of iodo-doxorubicin and doxorubicin in humans. Eur J Clin Pharmacol 39: 507-513

Olson RD, Mushlin PS (1990) Doxorubicin cardiotoxicity: Analysis of prevailing hypothesis. FASEB J 4: 3076-3086

Schaefer U, Schneider D, Rixen D, Neugebauer E (1998) Neutrophil adhesion to histamine stimulated cultured endothelial cells is primarily mediated via activation of phospholipase $\mathrm{C}$ and nitric oxide synthase isozymes. Inflamm Res 47: 256-264

Schwartz CL, Hobbie WL, Treusdel S, Constine LC, Clark EB (1993) Corrected QT interval prolongation in anthracycline-treated survivors of childhood cancer. J Clin Oncol 11: 1906-1910

Takacs IE, Matkovics B, Varga SI, Homolay P, Feher G, Seres T (1992) Study of the myocardial antioxidant defense in various species. Pharmacol Res 25: $177-178$

Van Acker FA, Van Acker SA, Kramer K, Haenen GR, Bast A, Van der Vijgh WJF (2000) 7-monohydoxyethylrutoside protects against chronic doxorubicin-induced cardiotoxicity when administered only once per week. Clin Cancer Res 6(4): 1337-1341

Van Acker SABE, Towart R, Husken BCP, De Jong J, Van der Vijgh WJF, Bast A (1993) The protective effect of Venoruton and its main constituents on acute doxorubicin-induced cardiotoxicity. Phlebology (Suppl. 1): $31-32$

Van Acker SA, Boven E, Kuiper K, Van den Berg D, Grimbergen JA, Kramer K, Bast A, Van der Vijgh WJF (1997) Monohydoxyethylrutoside, a dosedependent cardioprotective agent, does not affect the antitumor activity of doxorubicin. Clin. Cancer Res. 3: $1747-1754$

Van der Vijgh WJF, Maessen PA, Pinedo HM (1990) Comparative metabolism and pharmacokinetics of doxorubicin and $4^{\prime}$-epidoxorubicin in plasma, heart and tumor of tumor-bearing mice. Cancer Chemother. Pharmacol 26: 9-12

Verheul HMW, Jorna AS, Hoekman K, Broxterman HJ, Gebbink MFBG, Pinedo HM (2000) Vascular endothelial growth factor stimulated endothelial cells promote adhesion and activation of platelets. Blood 96: $4216-4221$ 